\title{
DUAL-FUNCTION RADAR-COMMUNICATIONS USING PHASE-ROTATIONAL INVARIANCE
}

\author{
Aboulnasr Hassanien, Moeness G. Amin, Yimin D. Zhang, and Fauzia Ahmad \\ Center for Advanced Communications, Villanova University, Villanova, PA 19085, USA \\ hassanien@ieee.org, \{moeness.amin,yimin.zhang, fauzia.ahmad\}@villanova.edu
}

\begin{abstract}
In this paper, we develop a new technique for dual-function radar-communications in a transmit multi-sensor array where information embedding is achieved using phase-rotational invariance. A sequence of $Q$ bits is first mapped into a dictionary of $2^{Q}$ phase rotations. Then, one pair of transmit orthogonal waveforms is used in tandem with $2^{Q}$ pairs of transmit beamforming weight vectors for embedding a certain entry of the phase-rotation dictionary during each radar pulse. The same pair of waveforms is used during all pulses while the pair of transmit beamforming weight vectors changes from pulse to pulse based on which entry of the phase-rotation dictionary is embedded. During each pulse, the receiver detects the embedded phase rotation and employ it to decipher the transmitted bit sequence. The proposed information embedding technique is angle-dependant and, therefore, the communication process is inherently secure against interception from directions other than the desired communication direction. The performance of the proposed technique is investigated in terms of the bit error rate (BER).
\end{abstract}

Index Terms - Dual-function radar-communications, phase-rotational invariance, communication security, bit error rate

\section{INTRODUCTION}

Efforts to address the concerns of increasing radio frequency spectrum congestion through co-existence and shared spectrum access of radar and wireless communication systems include cognitive radio [1], cognitive radar [2], as well as the cooperative operation of radar and communication systems [3]- [7]. Embedding information into radar emission as secondary to the primary radar function has been reported in a number of papers [8]- [11]. Recently, a dual-function radar-communications (DFRC) approach using time modulated arrays has been proposed [10]. In this approach, multiple sidelobe levels towards the communication direction are utilized for information embedding. More recently, a DFRC technique using sidelobe control in tandem with waveform diversity has been proposed where a set of $Q$ orthogonal waveforms are used for embedding a sequence of $Q$ bits of infor- mation [11]. The sidelobe levels (SLLs) towards the communication directions are controlled to have two distinct levels via designing two transmit beamforming weight vectors. However, the aforementioned two methods enable communications only towards directions that are located in the silelobe region where the radiated power is typically minimized from the source.

In this paper, we propose a new DFRC technique using phase-rotational invariance. In order to deliver a message of $Q$ bits per radar pulse, the corresponding entry of a phaserotation dictionary of size $K=2^{Q}$ is embedded in the radar emission. The communication receiver detects the embedded phase-rotation and, correspondingly, deciphers the transmitted communication message. During each pulse, one pair of transmit orthogonal waveforms is used in tandem with one pair of transmit beamforming weight vectors for embedding a certain phase rotation. The same pair of transmit orthogonal waveforms is transmitted during all radar pulses in order not to affect the coherent processing of the radar signals at the radar receiver. The pair of transmit beamforming weight vectors changes from pulse to pulse based on which entry of the phase-rotation dictionary is embedded during a certain pulse. Unlike previous DFRC methods which enable communications only towards directions located in the sidelobe regions, the proposed method enables communications towards any arbitrary direction, including directions located in the mainlobe of the radar operation. Moreover, the communication process is inherently secure against intercepts from directions other than the desired communication direction. In addition, the proposed method does not require phase synchronization as it does not require the estimation of the phase of the received signal, but rather it depends on the decoding of phaserotation between two independent virtual components of the received signal. The superiority of the proposed method over the recently developed methods in [10] and [11] is validated using simulation examples.

\section{SIGNAL MODEL}

We consider a radar system equipped with $M_{T}$ transmit antennas arranged as a uniform linear array and $M_{R}$ receive antennas arranged in an arbitrary linear shape. The primary 
radar operation of the system mandates focusing the transmit power within the main beam region while keeping the sidelobe levels of the transmit radiation pattern below a certain predetermined threshold. As a secondary function, we utilize one pair (or multiple pairs) of orthogonal waveforms in order to embed one (or several) data bits towards predetermined communication receivers with known directions. The $M_{T} \times 1$ baseband representation vector of the signals at the input of the transmit antennas is given by

$$
\mathbf{s}(t)=\mathbf{w} \psi(t)+\tilde{\mathbf{w}} \tilde{\psi}(t),
$$

where $t$ is the fast time index, $\psi(t)$ and $\tilde{\psi}(t)$ represent a pair of orthogonal waveforms, and $\mathbf{w}$ and $\tilde{\mathbf{w}}$ are the $M \times 1$ transmit beamforming weight vectors used to control the transmit power radiation patterns associated with $\psi(t)$ and $\tilde{\psi}(t)$, respectively.

Assuming that $L$ far-field targets are located within the radar main beam, the $M_{R} \times 1$ baseband representation of the signals at the output of the receive antenna arrays is given as

$$
\mathbf{x}(t ; \tau)=\sum_{l=1}^{L} \beta_{l}(\tau)\left(\mathbf{a}^{T}\left(\theta_{l}\right) \mathbf{s}(t ; \tau)\right) \mathbf{b}\left(\theta_{l}\right)+\mathbf{z}(t ; \tau),
$$

where $\tau$ is the slow-time index (i.e., pulse number), $\beta_{l}(\tau)$ is the $l$ th target reflection coefficient which obeys the Swirlling II target model, i.e., it remains constant during the entire radar pulse but changes from pulse to pulse. In addition, $\mathbf{a}(\theta)$ and $\mathbf{b}(\theta)$ are the $M_{T} \times 1$ and the $M_{R} \times 1$ steering vectors of the transmit and receive arrays, respectively, $\mathbf{z}(t ; \tau)$ is the $M_{R} \times 1$ vector of additive white Gaussian noise with zero mean and covariance $\sigma_{z}^{2} \mathbf{I}_{M_{R}},(\cdot)^{T}$ stands for the transpose, and $\mathbf{I}_{M_{R}}$ denotes the $M_{R} \times M_{R}$ identity matrix. It is worth noting that the processing of the radar received data can be performed directly on the $M_{R} \times 1$ data $\mathrm{x}(t ; \tau)$, i.e., without making use of the waveform diversity. In such a case, pulse compression can be performed by matched-filtering the received data vector $\mathbf{x}(t ; \tau)$ to the waveform $\Psi(t)=\psi(t)+\tilde{\psi}(t)$. Alternatively, incorporating a preprocessing step via matched-filtering the received data to the orthogonal transmit waveforms may lead to improved radar operation. In the latter case, the received data vector $\mathbf{x}(t ; \tau)$ is separately matched-filtered to $\psi(t)$ and $\tilde{\psi}(t)$, yielding two virtual data vectors of the same dimensionality as that of $\mathbf{x}(t ; \tau)$.

For the secondary function (i.e., communications function) of the proposed system, consider $J$ communication receivers located at arbitrary spatial directions known to the transmitter. The orthogonal waveform dictionary used at the transmitter is assumed to be known to each communication receiver. The operations of the individual communication receivers are independent from each others. In this respect, the received signal model, given below, is assumed for all receivers. The baseband representation of the signal at the output of the communication receiver is given as,

$$
x_{c}(t ; \tau)=G_{c} \alpha_{c}(\tau) \mathbf{a}^{T}\left(\theta_{c}\right) \mathbf{s}(t ; \tau)+n_{c}(t ; \tau),
$$

where $G_{c}$ is the coherent processing gain of the array in case multiple receive antennas are used at the receiver, $\alpha_{c}$ is the propagation coefficient of the received signal which summarizes the propagation environment between the transmit array and the communication receiver during the $\tau$ th pulse, and $n_{c}(t ; \tau)$ is the additive white Gaussian noise with zero mean and variance $\sigma_{c}^{2}$. Substituting (1) in (3) and matched-filtering the received data to $\psi(t)$ yields

$$
y(\tau)=\tilde{\alpha}_{c}(\tau)\left(\mathbf{w}^{H} \mathbf{a}\left(\theta_{c}\right)\right)+n(\tau),
$$

where $\tilde{\alpha}_{c}(\tau) \triangleq \alpha_{c}(\tau) G_{c}$ is the complex amplitude of the signal at the output of the matched-filter, $(\cdot)^{H}$ stands for the Hermitian, and $n(\tau)$ is white Gaussian noise term at the output of the matched filter with zero-mean and variance $\sigma_{c}^{2}$. Similarly, matched-filtering $x_{c}(t ; \tau)$ to the waveform $\tilde{\psi}(t)$ yields

$$
\tilde{y}(\tau)=\tilde{\alpha}_{c}(\tau)\left(\tilde{\mathbf{w}}^{H} \mathbf{a}\left(\theta_{c}\right)\right)+\tilde{n}(\tau),
$$

where $\tilde{n}(\tau)$ is white Gaussian noise term at the output of the matched filter with zero-mean and variance $\sigma_{c}^{2}$.

It is worth noting that, if $\mathbf{w}$ and $\tilde{\mathbf{w}}$ are appropriately designed such that the condition $\left|\mathbf{w}^{H} \mathbf{a}\left(\theta_{c}\right)\right|=\left|\tilde{\mathbf{w}}^{H} \mathbf{a}\left(\theta_{c}\right)\right|$ is satisfied, then $y(\tau)$ and $\tilde{y}(\tau)$ are guaranteed to enjoy the socalled rotational invariance property, where $|\cdot|$ stands for the magnitude of a complex number. This property means that the noise-free version of $y(\tau)$ equals the noise-free version of $\tilde{y}(\tau)$ up to some phase rotation $\phi_{c}$. This phase rotation can be expressed as

$$
\phi_{c}=\angle \frac{\mathbf{w}^{H} \mathbf{a}\left(\theta_{c}\right)}{\tilde{\mathbf{w}}^{H} \mathbf{a}\left(\theta_{c}\right)} .
$$

Information embedding can be achieved by controlling the value of the phase rotation at the transmit side and employing a phase decoder at the communications receiver.

\section{PROPOSED INFORMATION EMBEDDING TECHNIQUE}

We start with a principal transmit beamforming weight vector w which satisfies a certain desired transmit power radiation pattern. Sophisticated methods for designing a single transmit beamforming weight vector that achieves a desired pattern with ripple and transition band properties have been reported in the literature, e.g., [13]- [17]. The $M \times 1$ principal weight vector can be used to generate a population of $2^{M-1}$ weight vectors of the same dimensionality which have the same transmit power radiation pattern as that of w [13], [14]. The aforementioned population, denoted as $\mathbf{W}=\left\{\mathbf{w}_{1}, \ldots, \mathbf{w}_{2^{M-1}}\right\}$, can be obtained by viewing the principal weight vector as a polynomial of order $M-1$ with $M-1$ roots denoted as $r_{i}, i=1, \ldots, M-1$. Note that reflecting each root against the unit circle does not change the magnitude of the beampattern. At most $2^{M-1}$ different 
polynomials of the same order can be constructed, depending on the employed $M-1$ combinations of the roots $r_{i}$ (or $\left.1 / r_{i}^{*}\right), i=1, \cdots, M-1$ for polynomial construction, where $(\cdot)^{*}$ stands for the conjugate.

During each radar pulse, a $Q$-bit information message composed of 1's and 0's is denoted as the binary sequence $B_{q}, q=1, \ldots, Q$. In order to embed such a message in the transmitted signal, we build a transmit beamforming weight vector dictionary of $K=2^{Q}$ pairs, denoted as $\left\{\mathbf{w}_{1}, \tilde{\mathbf{w}}_{1}\right\}, \ldots,\left\{\mathbf{w}_{K}, \tilde{\mathbf{w}}_{K}\right\}$, which can be chosen from the population $\mathbf{W}$. Let $\phi_{1}, \ldots, \phi_{K}$ be the $K$ dimensional phaserotation alphabet associated with the $K$ pairs, that is

$$
\phi_{k}=\angle \frac{\mathbf{w}_{k}^{H} \mathbf{a}\left(\theta_{c}\right)}{\tilde{\mathbf{w}}_{k}^{H} \mathbf{a}\left(\theta_{c}\right)}, \quad k=1, \ldots, K .
$$

Only one of the $K$ phase-rotational code is embedded during each radar pulse to deliver the $Q$ bits of information. Assume that the $k$ th symbol $\phi_{1}$ is required to be embedded during the $\tau$ th radar pulse. This can be achieved by casting the transmit signal vector as

$$
\mathbf{s}(t ; \tau)=\mathbf{w}_{k} \psi(t)+\tilde{\mathbf{w}}_{k} \tilde{\psi}(t)
$$

Similar to phase modulation in wireless communications, either binary or Gray code can be used for information embedding. However, in the proposed method, the information is represented in the form of the phase difference between two separate waveforms rather than the phase of a single waveform, making the detection easier and more robust.

Adopting the above transmit signal model, the signal at the output of the communication receiver is given as

$$
\begin{aligned}
x_{c}(t ; \tau)= & \tilde{\alpha}_{c}(\tau)\left(\mathbf{w}_{k}^{H} \mathbf{a}\left(\theta_{c}\right) \psi(t)\right. \\
& \left.+\tilde{\mathbf{w}}_{k}^{H} \mathbf{a}\left(\theta_{c}\right) \tilde{\psi}(t)\right)+n_{c}(t ; \tau) .
\end{aligned}
$$

The signal components associated with $\psi(t)$ and $\tilde{\psi}(t)$ at the output of the communication receiver matched filter can be restated, respectively, as

$$
\begin{aligned}
& y(\tau)=\tilde{\alpha}_{c}(\tau) \mathbf{w}_{k}^{H} \mathbf{a}\left(\theta_{c}\right)+n(\tau), \\
& \tilde{y}(\tau)=\tilde{\alpha}_{c}(\tau) \tilde{\mathbf{w}}_{k}^{H} \mathbf{a}\left(\theta_{c}\right)+\tilde{n}(\tau) .
\end{aligned}
$$

Therefore, the phase rotation embedded in the received signal can be estimated as

$$
\hat{\phi}(\tau)=\angle \frac{y(\tau)}{\tilde{y}(\tau)} .
$$

The actual sequence of bits embedded can be determined by comparing $\hat{\phi}(\tau)$ obtained from (12) to the phase-rotation dictionary (7).

\section{SIMULATION RESULTS}

In the simulations we consider a uniform linear transmit array consisting of $M=10$ antennas spaced half a wavelength apart.

Example 1. In the first example, we investigate the possibility of realizing multiple pairs of transmit beamforming weight vectors which have a certain desired transmit power distribution pattern as well as a desired phase-rotation dictionary. We assume that the main radar operation takes place

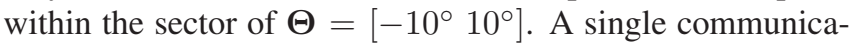
tion direction towards the spatial direction $\theta_{c}=-50^{\circ}$ is assumed. We design the principal transmit beamforming weight vector by solving the following optimization problem

$$
\begin{gathered}
\min _{\mathbf{w}} \max _{\theta_{i}}\left|\mathbf{w}^{H} \mathbf{a}\left(\theta_{i}\right)-e^{-j \pi \sin \theta_{i}}\right|, \theta_{i} \in \mathbf{\Theta}, i=1, \ldots, I \\
\text { s.t. }\left|\mathbf{w}^{H} \mathbf{a}\left(\theta_{p}\right)\right| \leq \varepsilon, \quad \theta_{p} \in \overline{\mathbf{\Theta}}, p=1, \ldots, P, \\
\left|\mathbf{w}^{H} \mathbf{a}\left(\theta_{c}\right)\right|=\varepsilon_{c}
\end{gathered}
$$

where $\overline{\boldsymbol{\Theta}}$ represents the out-of-sector region, $\theta_{i}, i=1, \ldots, I$, and $\theta_{p}, p=1, \ldots, P$, are discrete grids of angles used to approximate $\Theta$ and $\bar{\Theta}$, respectively, and $\varepsilon$ is the parameter that defines the highest allowable sidelobe level. It is assumed that the radar operation requires the power level emitted in the sidelobe areas to be at least $20 \mathrm{~dB}$ lower than the mainlobe and, therefore, $\varepsilon=\sqrt{0.01}$ is chosen. Note that the constraint (15) determines the SLL towards the communication direction which is chosen to be equal to the highest allowable level, i.e., $\varepsilon_{c}=\sqrt{0.01}$ is used. The values $I=200$ and $P=140$ are used to approximate the desired sector and the out-of-sector regions, respectively. The optimization problem (13)-(15) is convex and can be solved efficiently using the interior point method [12]. Fig. 1 shows the transmit power distribution versus the spatial angle for the principal transmit beamforming weight vector.

The principal weight vector is used to generate a population of $2^{M-1}=512$ weight vectors which have exactly the same transmit power patterns. The population is used to build 256 pairs of vectors and the phase rotations associated with the communication direction $\theta_{c}=-50^{\circ}$ for all available pairs are plotted in Fig. 2. The figure shows that the available phase-rotations cover the entire phase domain between $0^{\circ}$ and $360^{\circ}$. This enables choosing a suitable phaserotation dictionary of size $2^{Q}$. For example, if $Q=2$ bit is required to be embedded then a phase-rotation dictionary of size $K=4$ is needed. One realization for this case is shown as the red circles in Fig. 2. It can be observed from the figure that the $K=4$ phase-rotation dictionary is almost uniformly distributed on the unit circle, resulting in better probability of detection at the receiver.

Example 2. In the second example, we investigate the performance of the proposed method in terms of the bit error rate (BER). We also evaluate how secure the communication process is by computing the BER at directions other than the 


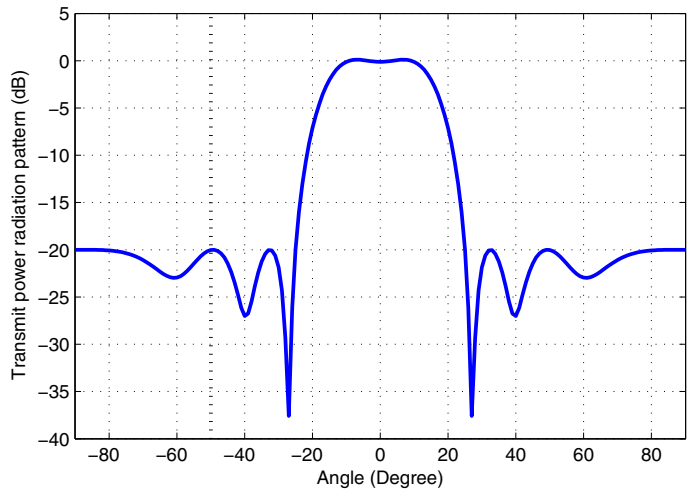

Fig. 1. Transmit power distribution versus spatial angle; first example.

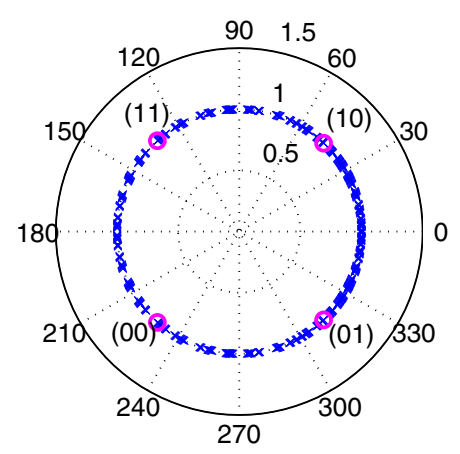

Fig. 2. Phase-rotation distribution versus spatial angle for a population of 256 pairs of transmit beamforming weight vectors; first example.

indented communication direction. The performance of the proposed method is compared with the techniques proposed in [10] and [11]. We assume that $Q=2$ bits of information are needed to be transmitted during every radar pulse. For the proposed method, we use the same parameters from the previous example. The phase-rotation dictionary of dimension $K=4$, which corresponds to the red circles in Fig. 2, is used. Note that the method described in [10] does not employ waveform diversity. Instead, it employs a single waveform in tandem with $2^{Q}$ SSLs towards the communication direction to deliver $Q$ bits of information. To deliver a $Q=2$ bit message, the method in [10] uses four transmit beamformers with four distinct SSLs towards the communication direction. These waveforms can be designed by solving (13)-(15) for $\varepsilon_{c}=\sqrt{0.01}, \sqrt{0.0033}, \sqrt{0.0066}$, and $\sqrt{0.0001}$, respectively. For the method of [11], two transmit weight vectors are used along with two orthogonal waveforms. In particular, the two weight vectors associated with $\varepsilon_{c}=\sqrt{0.01}$ and $\sqrt{0.0001}$ are used to implement the method of [11].

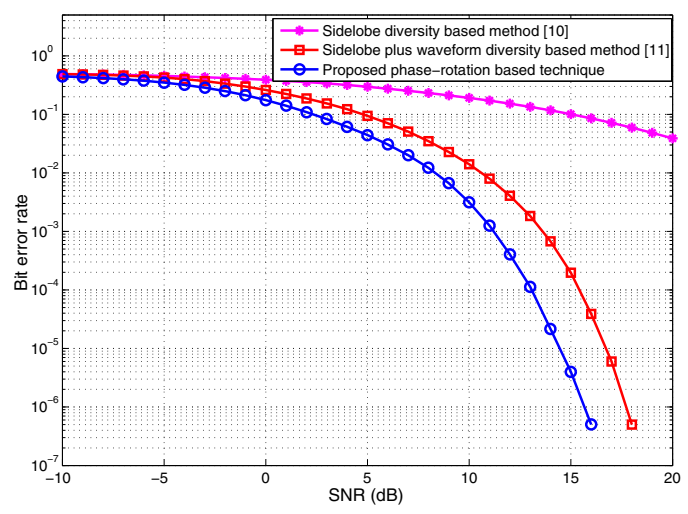

Fig. 3. BER versus SNR; second example.

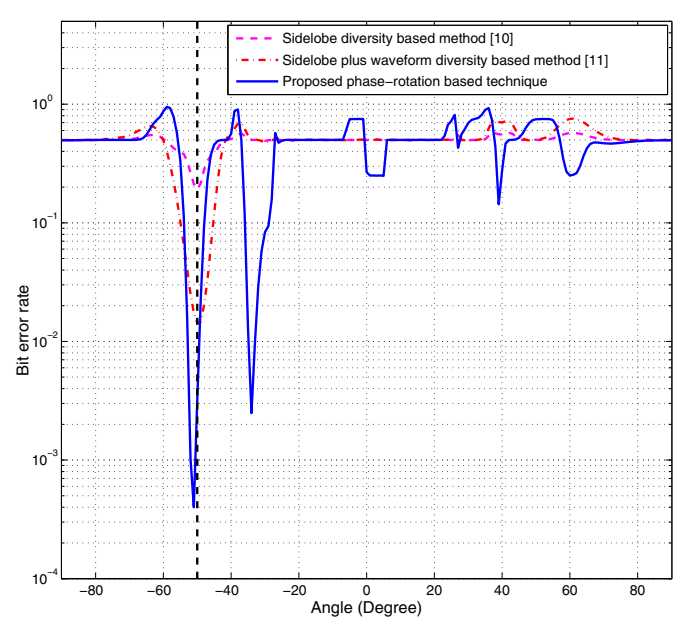

Fig. 4. BER versus spatial angle of the communication receiver; second example.

To compute the BER, $10^{6}$ sequences (two bits each) are transmitted. The propagation coefficient $\alpha_{c}$ is modeled as a random variable with constant unity mangnitude and uniformly distributed random phase and the communication receive gain is chosen as $G_{c}=3.16$. Fig. 3 shows the BERs for the three methods tested versus the signal-to-noise ratio (SNR). It is clear that the proposed technique achieves a superior BER performance as compared to the methods of [10] and [11]. Note that, unlike the methods of [10] and [11], the proposed method enables communications within the main radar beam.

Finally, Fig. 4 depicts the BER versus the spatial angle with the SNR fixed to $10 \mathrm{~dB}$ for all methods. It can be observed from the figure that the proposed method has better BER towards the communication direction as compared to the methods of [10] and [11]. It can also be confirmed from the 
figure that, for all methods, the BER is very high for almost all directions other than the communication direction of interest. This means that all methods have inherent security against information interception from directions other than the communication direction. Although the figure shows that, for the proposed method, the information message can be intercepted with a relatively low BER from the direction $-34^{\circ}$, robust transmit beamforming design with robustness against eavesdropping can be employed to improve the communication security. This will be investigated further in the near future.

\section{CONCLUSION}

A new technique for dual-function radar-communication system using phase-rotation invariance has been developed. In order to deliver a sequence of $Q$ bits per radar pulse, a pair of orthogonal waveforms is needed in tandem with $2^{Q}$ appropriate pairs of transmit beamforming weight vectors. The communication receiver detects the phase-rotation between the signal components associate with the two waveforms and maps it to an information sequence. As compared to previous methods which enable communications only towards directions located in the sidelobe region, the proposed technique permits information delivering towards arbitrary communication directions including directions located in the mainlobe region. The communication process is inherently secure against interception from directions other than the presumed communication directions. The effectiveness of the proposed technique and its superiority over existing techniques were verified through simulations examples.

\section{REFERENCES}

[1] A. J. Goldsmith and L. J. Greenstein, Principles of Cognitive Radio. Cambridge University Press, 2012.

[2] S. Haykin, Cognitive dynamic systems: Perception-Action Cycle, Radar and Radio. Cambridge University Press, 2012.

[3] H. Griffiths, S. Blunt, and L. Chen, "Challenge problems in spectrum engineering and waveform diversity," in Proc. IEEE Radar Conf. (RadarCon 2013), Ottawa, ON, Canada, Apr.May 2013, pp. 1-5.

[4] D. W. Bliss, "Cooperative radar and communications signaling: The estimation and information theory odd couple," in Proc. IEEE Radar Conf. (RadarCon 2014), Cincinnati, OH, May 2014, pp. 50-55.

[5] M. Jamil, H. Zepernick, and M. I. Pettersson, "On integrated radar and communication systems using Oppermann sequences," in Proc. IEEE Military Communications Conf. (MILCOM 2008), Nov. 2008, pp. 1-6.

[6] S .C. Surender, R. M. Narayanan, C. R. Das, "Performance analysis of communications \& radar coexistence in a covert UWB OSA system," in Proc. IEEE Global Commun. Conf. (GLOBECOM 2010), Miami, FL, Dec. 2010, pp. 1-5.

[7] Y. L. Sit, C. Sturm, L. Reichardt, T. Zwick, and W. Wiesbeck, "The OFDM joint radar-communication system: An overview," in Proc. Int. Conf. Advances in Satellite and Space Communications (SPACOMM 2011), Budapest, Hungary, Apr. 2011, pp. 69-74.

[8] R. M. Mealey, "A method for calculating error probabilities in a radar communication system," IEEE Trans. Space Electronics and Telemetry, vol. 9, no .2, pp. 37-42, June 1963.

[9] S. D. Blunt, P. Yatham, and J. Stiles, "Intra-pulse radarembedded communications," IEEE Trans. Aerospace and Electronic Systems, vol. 46, no. 3, pp. 1185-1200, July 2010.

[10] J. Euziere, R. Guinvarc'h, M. Lesturgie, B. Uguen, and R. Gillard, "Dual function radar communication timemodulated array," in Proc. Int. Radar Conf., Lille, France, Oct. 2014.

[11] A. Hassanien, M. G. Amin, Y. D. Zhang, and F. Ahmad, "A dual function radar-communications system using sidelobe control and waveform diversity," in Proc. 2015 IEEE Int. Radar Conf. (RadarCon 2015), Arlington, VA, May 2015.

[12] S. Boyd and L. Vandenberghe, Convex Optimization. Cambridge University Press, 2009.

[13] A. Khabbazibasmenj, A. Hassanien, and S. A. Vorobyov, "How many beamforming vectors generate the same beampattern?" arXiv preprint, arXiv:1402.1682, 2014.

[14] A. Hassanien, S. A. Vorobyov, and A. Khabbazibasmenj, "Transmit radiation pattern invariance in MIMO radar with application to DOA estimation," IEEE Signal Process. Lett., vol. 22, pp. 1609-1613, Oct 2015.

[15] Z. L. Yu, W. Ser, M. H. Er, Z. Gu, and Y. Li, "Robust adaptive beamformers based on worst-case optimization and constraints on magnitude response," IEEE Trans. Signal Process., vol. 57, no. 7, pp. 2615-2628, July 2009.

[16] A. Hassanien, M. G. Amin, Y. D. Zhang, and F. Ahmad, "Capon-based single snapshot DOA estimation in monostatic MIMO Radar," in Proc. Symposium SPIE Sensing Technology + Applications, Baltimore, MD, Apr. 2015.

[17] A. Hassanien, M. G. Amin, Y. D. Zhang, and F. Ahmad, "High-resolution single-snapshot DOA estimation in MIMO radar with colocated antennas," in Proc. 2015 IEEE Int. Radar Conf. (RadarCon 2015), Arlington, VA, May 2015. 